Check for updates

Cite this: RSC Adv., 2017, 7, 19674

Received 21st February 2017

Accepted 25th March 2017

DOI: $10.1039 / c 7 r a 02186 k$

rsc.li/rsc-advances

\section{Functionalization of $\mathrm{BaTiO}_{3}$ nanoparticles with electron insulating and conducting organophosphazene-based hybrid materials $\uparrow$}

\author{
George S. Pappas, (D) ${ }^{a}$ Chaoying Wan, (D) *a Chris Bowen, (D) ${ }^{b}$ David M. Haddleton (1D) ${ }^{c}$ \\ and Xiaobin Huang ${ }^{\text {d }}$
}

Novel core-shell structured organophosphazene (OPZ) coated $\mathrm{BaTiO}_{3}$ nanoparticles $\left(\mathrm{OPZ}\left(\mathrm{BaTiO}_{3}\right)\right.$ were produced via a facile and rapid one-step nucleophilic substitution reaction in ambient conditions. The morphology, structure and textural properties of the core-shell nanoparticles were analysed via electron microscopy, spectroscopy, thermogravimetry and porosimetry, and the dielectric properties were evaluated by impedance spectroscopy. The thickness of the cross-linked OPZ shell was readily tailored by varying the weight ratio of the $\mathrm{OPZ}$ monomers to $\mathrm{BaTiO}_{3}$, which in turn affected the relative permittivity and the frequency dependence of the $\mathrm{OPZ} / \mathrm{BaTiO}_{3}$ particles. A subsequent carbonisation treatment of the $\mathrm{OPZ}\left(\mathrm{aBaTiO}_{3}\right.$ at $700{ }^{\circ} \mathrm{C}$ transformed the polymeric OPZ shell to a microporous carbonaceous shell, which dramatically increased the electrical conductivity of the particles. Organophosphazene chemistry offers a facile route to functionalise $\mathrm{BaTiO}_{3}$ nanoparticles without any pre-treatment, and generate a range of core-shell $\mathrm{BaTiO}_{3}$ nanoparticles with tailored dielectric and electrically conductive properties that can be used as active fillers for polymer based nanocomposites and energy storage applications. The effectiveness and advantages of OPZ chemistry over other reported methods in forming core-shell particles are demonstrated.

\section{Introduction}

Ferroelectric poly(vinylidenefluoride) (PVDF) and its copolymers, such as poly(vinylidenefluoride-co-trifluoroethylene) (PVDF- $c o$ $\mathrm{TrFE}$ ), are attractive materials for applications in dielectric devices due to their high dielectric strength $\left(E_{\mathrm{b}} \sim 4 \mathrm{MV} \mathrm{cm}^{-1}\right)$ and convenient processability. However, their energy storage is limited by their low relative dielectric constant $\left(\varepsilon_{\mathrm{r}} \sim 10\right)^{1,2}$ In order to further enhance the relative permittivity and energy density of PVDF-based composites, ferroelectric ceramics, such as $\mathrm{BaTiO}_{3}$, with a significantly higher relative permittivity $\left(\varepsilon_{\mathrm{r}} \sim\right.$ 1700) has been utilised as a dielectric additive., ${ }^{3,4}$ The incorporation of $\mathrm{BaTiO}_{3}$ nanoparticles into a polymer matrix, usually involves a surface modification process in order to prevent agglomeration and phase separation, and to increase their interfacial interaction with the polymers. A variety of surface-

${ }^{a}$ International Institute for Nanocomposites Manufacturing (IINM), WMG, University of Warwick, CV4 7AL, UK. E-mail: chaoying.wan@warwick.ac.uk

${ }^{b}$ Materials and Structures Centre, Department of Mechanical Engineering, University of Bath, BA2 7AY, UK

'Department of Chemistry, University of Warwick, CV4 7AL, UK

${ }^{d}$ School of Aeronautics and Astronautics, Shanghai Jiao Tong University, 200240, P. R. China

$\dagger$ Electronic supplementary information (ESI) available. See DOI: $10.1039 / \mathrm{c} 7 \mathrm{ra} 02186 \mathrm{k}$ treatment approaches have been investigated which include: introduction of surface hydroxyls by chemical etching of $\mathrm{BaTiO}_{3}$ particles with $\mathrm{H}_{2} \mathrm{O}_{2},{ }^{5}$ chemical modification with pentafluorobenzyl phosphonic acid ${ }^{6}$ or fluorosilane, ${ }^{7}$ and polymeric coatings such as polydopamine ${ }^{8}$ and polyaniline. ${ }^{9}$ Furthermore, polymer brushes grafted on $\mathrm{BaTiO}_{3}$ nanoparticles, have been synthesized by controlled free radical surface-initiated polymerization techniques such as the reversible addition-fragmentation chain transfer (RAFT), ${ }^{10,11}$ and the atom transfer radical polymerization (ATRP). ${ }^{12}$ Despite the promising results, the methods employed above involve extensive pre-treatment of the surface of $\mathrm{BaTiO}_{3}$ particle prior to further functionalisation. The existing processing routes therefore require multi-step reaction and purification procedures which leads to low production yield and high cost.

In addition to dielectric and ferroelectric ceramics, electrically conductive carbons can be used to enhance the relative permittivity of $\mathrm{PVDF} / \mathrm{BaTiO}_{3}$ composites while maintaining a low dielectric loss. ${ }^{13,14}$ Furthermore, the presence of $\mathrm{BaTiO}_{3}$ in these composite systems can also facilitate the dispersion of carbons in the polymer matrix, resulting in a higher interfacial interaction with the PVDF matrix. ${ }^{15}$ In such systems, the increased relative permittivity by the addition of conductive fillers, is attributed to the interfacial polarization resulting from the Maxwell-Wagner-Sillars (MWS) effect. ${ }^{16}$ Recently, the decoration of $\mathrm{BaTiO}_{3}$ with conductive graphitic carbon via 
a chemical vapour deposition (CVD) process, showed its beneficial role in the energy storage of PVDF-HFP composites. ${ }^{17}$

Here we have explored a facile core-shell approach to functionalise $\mathrm{BaTiO}_{3}$ nanoparticles through organophosphazene (OPZ) chemistry. A highly cross-linked OPZ shell is formed on the $\mathrm{BaTiO}_{3}$ surface via a one-step reaction at room temperature and ambient conditions. The formation mechanism of $\mathrm{OPZ}$ is based on the $\mathrm{S}_{\mathrm{N}} 2$ nucleophilic substation of hexachlorocyclotriphosphazene (HCCP) by a multifunctional aromatic compound to generate numerous primary oligomers, and simultaneous aggregation to form a stable and highly cross-linked structure..$^{18-20}$ The reaction takes place in the presence of an aprotic polar solvent (acetonitrile, tetrahydrofuran, etc.) and an excess amount of triethylamine (TEA) which traps the $\mathrm{Cl}^{-}$released from the substitution on the $\mathrm{P}$ atoms of the HCCP. The nature of the above reaction provides an efficient route for producing core-shell nanostructures by applying the same chemistry in the presence of a template. ${ }^{21}$ Furthermore, the OPZ shell can be transformed to an electrically conductive carbon shell by carbonisation under inert atmosphere at high temperatures. The produced carbon structure is highly microporous with small graphitic crystals in a "house of cards" arrangement and with a relatively high degree of heteroatom-doping provided from the monomers. ${ }^{22,23}$ The core-shell nanoparticles can readily serve as a dielectric or conductive additives for polymer-based nanocomposites and energy storage applications. In this context, coreshell $\mathrm{OPZ} / \mathrm{BaTiO}_{3}$ nanoparticles with tailored shell structure are studied. The structure and properties of the dielectric

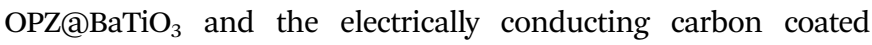
$\mathrm{BaTiO}_{3}\left(\mathrm{C} @ \mathrm{BaTiO}_{3}\right)$ are discussed.

\section{Experimental}

\subsection{Materials}

Hexachlorocyclotriphosphazene (HCCP, Sigma-Aldrich, UK), 4,4'-sulphonyldiphenol (BPS, Sigma-Aldrich, UK), triethylamine (TEA, Fisher Sci, UK), $\mathrm{CH}_{3} \mathrm{CN}$ were used as received. $\mathrm{BaTiO}_{3}$ (Sigma-Aldrich UK, cubic, $<50 \mathrm{~nm}$ ) particles were dispersed in acetonitrile $\left(\mathrm{CH}_{3} \mathrm{CN}\right)$ and sonicated for at least three hours before experiments. Acetone and distilled water were used for the purification of the products.

\subsection{Synthesis of core-shell OPZ@BaTio ${ }_{3}$ and $\mathrm{C} @ \mathrm{BaTiO}_{3}$}

Organophosphazene coated $\mathrm{BaTiO}_{3}$ nanoparticles were successfully prepared by the polycondensation reaction of HCCP and BPS in the presence of $\mathrm{BaTiO}_{3}$ nanoparticles. In a typical synthesis (OPZ@BaTiO $\left.{ }_{3}-1\right), 0.5 \mathrm{~g}$ of $\mathrm{BaTiO}_{3}$ were dispersed in $333 \mathrm{ml}$ of $\mathrm{CH}_{3} \mathrm{CN}$ by sonication for at least 3 hours. HCCP $(203 \mathrm{mg})$ and BPS $(463 \mathrm{mg})$ were added to the above mixture and left under sonication for $10 \mathrm{~min}$ before the flask was transferred on a magnetic stirrer. Under mild stirring, $5 \mathrm{ml}$ of TEA was added and the reaction mixture left for 20 hours. The solids were collected by centrifugation, washed $1 \times$ acetone and $2 \times$ acetone/water mixture and then dried in a vacuum oven at $50{ }^{\circ} \mathrm{C}$. Carbon coated $\mathrm{BaTiO}_{3}$ nanoparticles were obtained by

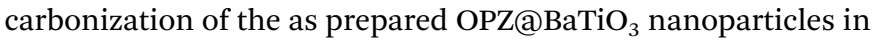
a tube furnace at $700{ }^{\circ} \mathrm{C}$ under $\mathrm{N}_{2}$ flow. The heating rate was
$2.5{ }^{\circ} \mathrm{C} \mathrm{min}{ }^{-1}$ and an isothermal step at $700{ }^{\circ} \mathrm{C}$ was maintained for 2 hours before the furnace physically let to cool down at room temperature.

\subsection{Synthesis of dense OPZ nanospheres}

Dense poly(cyclotriphosphazene-co-sulphonyl diphenol) nanospheres where synthesized in similar manner as the OPZ@BaTiO ${ }_{3}$ core-shell particles. In a $250 \mathrm{ml}$ round bottom flask containing $150 \mathrm{ml}$ of acetonitrile, HCCP $(170 \mathrm{mg})$ and BPS $(387 \mathrm{mg})$ were dissolved by sonication. The flask was transferred on a magnetic stirrer and TEA was added under a mild stirring speed $(400 \mathrm{rpm}, 3 \mathrm{~cm}$ stirring bar). The particle formation was observed in less than 10 seconds after the addition of the base and the mixture was left under continuous stirring for 3 hours. The OPZ nanospheres were collected by centrifugation at $8000 \mathrm{rpm}$, washed once with acetone and twice with acetone/water mixture and then dried in vacuum oven at $50{ }^{\circ} \mathrm{C}$. This sample was used for the TGA comparison with the OPZ@BaTiO ${ }_{3}$.

\subsection{Characterization}

Field emission gun-scanning electron microscopy (FEG-SEM) and X-ray electron dispersive spectroscopy analysis (EDS) were performed on a Zeiss SIGMA SEM. AuPd sputtering was applied to the samples before observation. The carbonized samples did not require any preparation due to their high electrical conductivity. Transmission electron microscopy (TEM) micrographs were obtained on JEOL 2000FX operated at $200 \mathrm{kV}$. Raman spectra were recorded on Renishaw inVia spectrometer equipped with $514 \mathrm{~nm}$ and 633 laser-lines. Thermogravimetric analysis curves were obtained with a Mettler Toledo instrument in the temperature range $25-700{ }^{\circ} \mathrm{C}$ at a heating rate of $10{ }^{\circ} \mathrm{C}$ $\min ^{-1}$ under $\mathrm{N}_{2}$ gas flow $\left(100 \mathrm{ml} \mathrm{min}^{-1}\right)$. The weight percentage of the carbon shell was calculated from TGA measurements under air flow. Nitrogen adsorption-desorption isotherms were obtained on a Quadrasorb Evo Analyser (Quantachrome). The specific surface area of the $\mathrm{OPZ} @ \mathrm{BaTiO}_{3}$ samples was calculated by the Brunauer-Emmett-Teller (BET) equation applied on the linear part of the adsorption branch $\left(0.05-0.30 P / P_{0}\right)$ and the pore size distribution was obtained by the Barrett-JoynerHalenda (BJH) method applied on the desorption branch data. For the $\mathrm{C} @ \mathrm{BaTiO}_{3}$ samples the specific surface area was calculated by the $t$-plot method and the pore size distribution by Nonlocal Density-Functional Theory (NLDFT) and applying the 'slit-like pores' model of carbon. The samples were degassed at $100{ }^{\circ} \mathrm{C}$ for 19 hours under high vacuum before measurements. The X-ray diffraction (XRD) patterns were recorded on a Panalytical Empyrean diffractometer with a $\operatorname{CoK} \alpha$ radiation source (1.7902 $\mathrm{A})$ operated at $45 \mathrm{kV}$ and $40 \mathrm{~mA}$. The scanning step width was $0.013^{\circ} 2 \theta$. X-ray Photoelectron Spectroscopy measurements (XPS) were contacted on a Kratos Axis Ultra DLD spectrometer using monochromatic Al $\mathrm{K} \alpha$ source $(h \nu=1486.6 \mathrm{eV})$. Survey spectra $(1200-0 \mathrm{eV})$ were collected with a pass energy of $160 \mathrm{eV}$, while the high-resolution spectra were obtained using a $20 \mathrm{eV}$ pass energy with a resolution approximately $0.4 \mathrm{eV}$. Peak fitting was performed on CasaXPS software, using mixed Gaussian- 
Lorentzian (Voigt) line shapes and Shirley backgrounds. For electrical measurements, pressed and electroded pellets of the powders were characterised; samples were $5 \mathrm{~mm}$ in diameter and typically 1-2 mm thick. Samples were dried for $24 \mathrm{~h}$ prior to electrical measurement to remove any adsorbed moisture. Electrical characterisation was undertaken by measurement of the real and imaginary impedance using a Solatron 1260 impedance analyzer with a Solatron 1296 dielectric interface at $0.1 \mathrm{~V}_{\mathrm{rms}}$ at frequencies from $1 \mathrm{~Hz}$ to $1 \mathrm{MHz}$. The ac conductivity, $\sigma$, was calculated using,

$$
\sigma=\frac{Z^{\prime}}{Z^{\prime 2}+Z^{\prime 2}} \frac{t}{A}
$$

where $Z^{\prime}$ and $Z^{\prime \prime}$ are the real and imaginary parts of the impedance, $A$ is the area of the sample and $t$ is the sample thickness. The relative permittivity (dielectric constant), $\varepsilon$, was calculated using,

$$
\varepsilon=\frac{-Z^{\prime \prime}}{Z^{\prime 2}+Z^{\prime \prime 2}} \cdot \frac{t}{\omega A \varepsilon_{0}}
$$

where $\omega$ is the angular frequency $(2 \pi f), f$ is frequency and $\varepsilon_{0}$ is the permittivity of free space. The phase angle $(\theta)$ between current and voltage and loss tangent $(\tan \delta$ ) was determined from,

$$
\begin{aligned}
& \theta=\tan ^{-1}\left(Z^{\prime \prime} / Z^{\prime}\right) \\
& \tan \delta=-Z^{\prime} / Z^{\prime \prime}
\end{aligned}
$$

\section{Results and discussion}

\subsection{Morphology and microstructure characterization}

Poly(organophosphazene)-coated dielectric nanoparticles

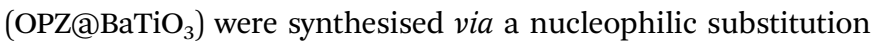
reaction between $\mathrm{HCCP}$ and $\mathrm{BPS}$ in the presence of $\mathrm{BaTiO}_{3}$ nanoparticles. Before the synthesis, the as-purchased commercial $\mathrm{BaTiO}_{3}$ powders consisted of micron-sized $(\sim 20 \mu \mathrm{m})$ spherical agglomerates were broken down and dispersed under extensive sonication in $\mathrm{CH}_{3} \mathrm{CN}$. The high polarity index of solvent was beneficial for: (i) assisting the dispersion and stabilization of the $\mathrm{BaTiO}_{3}$ nanoparticles in the solution, and (ii) promoting the nucleophilicity and therefore the reactivity of the BPS towards the $\mathrm{P}$ atoms of the HCCP. The previously described reaction mechanism for the formation of OPZ materials, can be also extended to the synthesis of core-shell particles; in the presence of $\mathrm{BaTiO}_{3}$ nanoparticles (or any other sub-micron particles), the primary HCCP-BPS oligomers formed the initial nuclei, which subsequently adsorbed on the surface of the $\mathrm{BaTiO}_{3}$ driven by the tendency to minimize their high free energy. Thus, there is no requirement for functional groups on the surface of the template nanoparticles, which is one the advantages of the current chemical method. After this stage, more HCCP and BPS monomers and/or HCCP-BPS oligomers, were diffused onto the coated $\mathrm{BaTiO}_{3}$ surface where the substitution reaction further took place and led to the growth of a cross-linked OPZ shell (Fig. 1).

OPZ@BaTiO ${ }_{3}$ nanoparticles with different shell-thicknesses were prepared by varying the weight ratio of the monomers to $\mathrm{BaTiO}_{3}$ from $0.25: 1$ to $4: 1$, while maintaining a constant molar ratio of the monomers of BPS to HCCP at $3: 1$ (Table $\mathrm{S} 1 \dagger)$. Fig. 2 shows the SEM images of the bare $\mathrm{BaTiO}_{3}$ nanoparticles and the core-shell OPZ@BaTiO ${ }_{3}$ particles prepared at different $[\mathrm{HCCP}-\mathrm{BPS}] /\left[\mathrm{BaTiO}_{3}\right]$ weight ratios. The shell thickness increased with an increase of the monomer concentration, and the shape of the particles became more regular as they grow to the sub-micron scale (Fig. 2e). The EDX spectrum revealed the elements existed in the core-shell structure (Fig. 2f) and the chemical composition of all the samples is summarized in Table $\mathrm{S} 2 . \dagger$ The ability to modify the $\mathrm{BaTiO}_{3}$ surface was confirmed for all the samples and no free $\mathrm{BaTiO}_{3}$ particles or dense OPZ spheres were observed; this result demonstrates that the presented OPZ chemistry can be applied to a wide range of [monomers]/[template] ratios and template concentrations without the need of extensive synthesis optimization. The absence of $\mathrm{BaTiO}_{3}$-free OPZs particles indicated that the nucleation and growth steps followed the "LaMer mechanism" where the initial fast nucleation lowered the monomers' concentration below a critical point and no further formation of new primary nuclei took place. ${ }^{24}$ In addition, the HCCP concentration was relatively low in order to prevent any secondary OPZ-particle formation.

By taking advantage of the capability of the In-lens detector to collect secondary electrons (SE) over a wide range of energies,

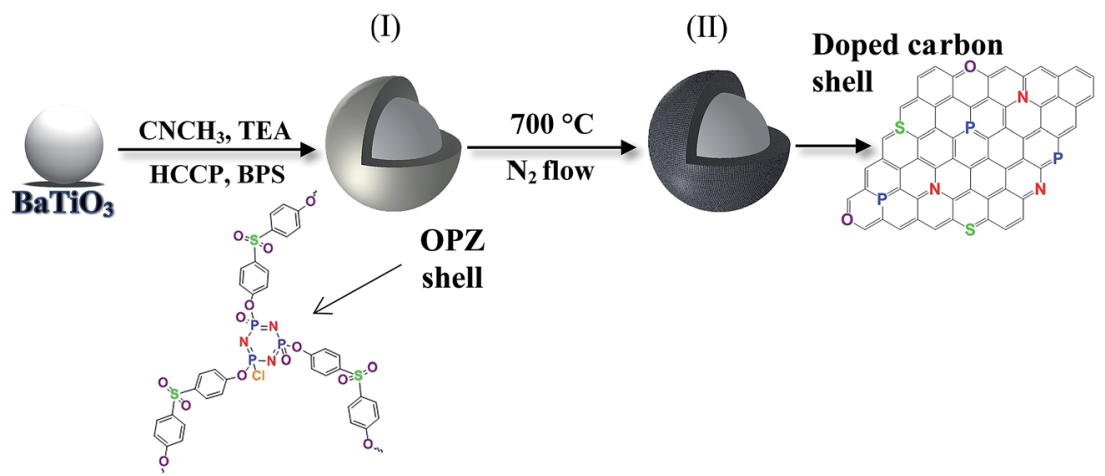

Fig. 1 Schematic illustration of the synthetic route for the production of (I) OPZ-coated $\mathrm{BaTiO}_{3}$ and (II) carbon-coated $\mathrm{BaTiO}$ - particles after a carbonization process. 

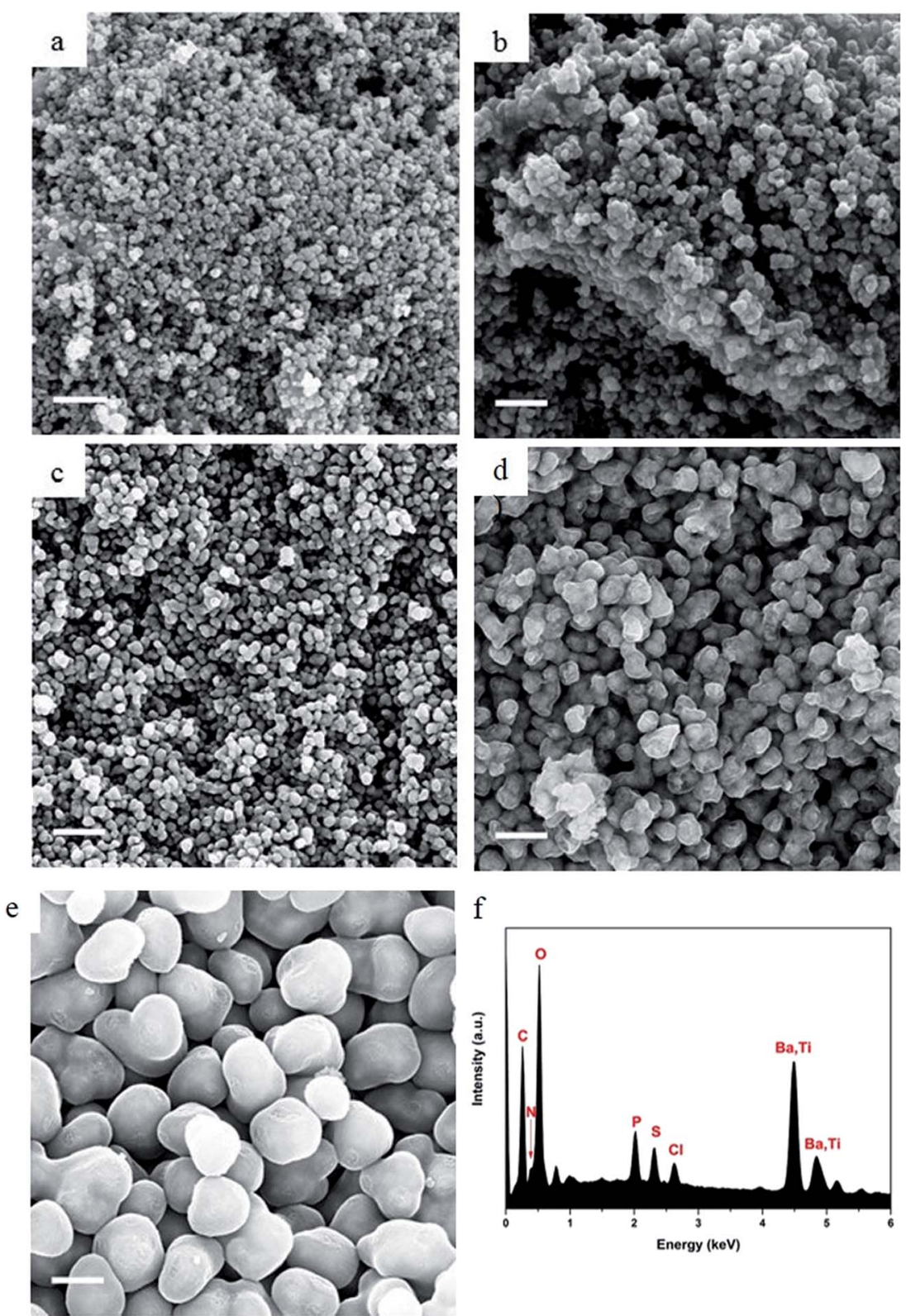

Fig. $2 \mathrm{SEM}$ images of (a) the commercial $\mathrm{BaTiO}_{3}$ particles after sonication, (b) OPZ@BaTiO $3-0.25$, (c) OPZ@BaTiO -0.5 , (d) OPZ@BaTiO $3-1$, (e) OPZ@BaTiO 3 -4. Scale bar at $500 \mathrm{~nm}$. (f) Representative spectra from EDX elemental analysis.

even at relatively low primary beam energy $(10 \mathrm{kV})$, the coreshell structure can be identified from the materials density difference (" $Z$ contrast"). ${ }^{25}$ The core $\left(\mathrm{BaTiO}_{3}\right)$ nanoparticles are almost visible under scanning electron microscopy (SEM) observation and this is more readily observed in the case of carbonized samples in Fig. 3 where the carbon shell has a more "transparent" appearance than the OPZ shell, giving the appearance of "TEM-like" images. This phenomenon is attributed to the conductivity of the carbon shell as well as to a lower density, compared to the OPZ shell, which allows the excited photoelectrons to escape from the structure more easily.

Fig. 3 shows the morphology of the carbonized samples C@BaTiO ${ }_{3}$, with shells of different thickness. The cross-linked OPZ shell was directly converted to a carbon shell doped with phosphorus, nitrogen, sulfur and oxygen as shown in the characteristic EDX spectrum (Fig. 3c, inset). After the carbonization process the overall size of the $\mathrm{C} @ \mathrm{BaTiO}_{3}$ particles decreased, since the conversion of the OPZ shell to carbon under $\mathrm{N}_{2}$ is followed by a significant mass loss, as shown from the TGA curves (Fig. 4). The onset decomposition temperature

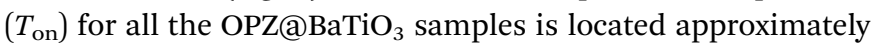
at $465{ }^{\circ} \mathrm{C}$. It is important to note that the thermogravimetric curves have a negative slope at $700{ }^{\circ} \mathrm{C}$, therefore the mass loss during carbonization in the furnace $\left(2 \mathrm{~h}\right.$ at $\left.700{ }^{\circ} \mathrm{C}\right)$ should be slightly higher than calculated from the TGA curves. The mass

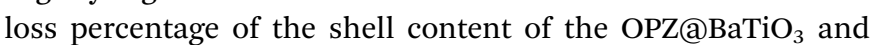
$\mathrm{C} @ \mathrm{BaTiO}_{3}$ core-shell structures was calculated from the $\mathrm{TGA}_{\text {air }}$ curves shown in Fig. S1(a and $\mathrm{b}) \dagger$ and all the results are 

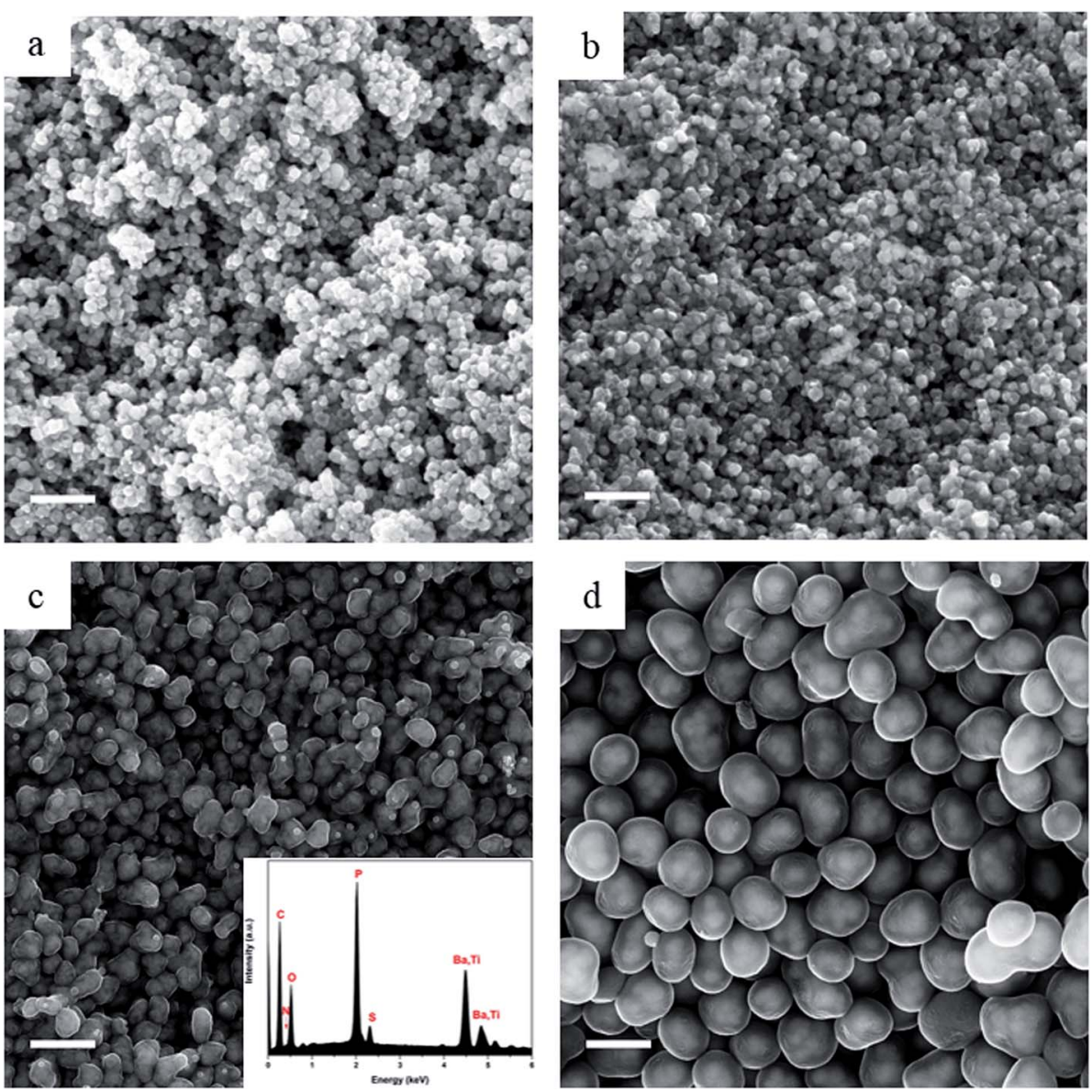

Fig. $3 \mathrm{SEM}$ images after carbonization at $700{ }^{\circ} \mathrm{C}$ of (a) $\mathrm{CaBaTiO}_{3}-0.25$, (b) $\mathrm{C}\left(\mathrm{BBaTiO}_{3}-0.5\right.$, (c) $\mathrm{C}(\mathrm{BBaTiO}-1$, and (d) $\mathrm{C}(\mathrm{BaTiO}-4$. Scale bar at $500 \mathrm{~nm}$.

summarized in Table 1. The $\mathrm{TGA}_{\mathrm{air}}$ of the OPZ@BaTiO ${ }_{3}$ showed an initial steep mass loss at $475^{\circ} \mathrm{C}$ due to the decomposition of the organic part (BPS) of the nanospheres and the process

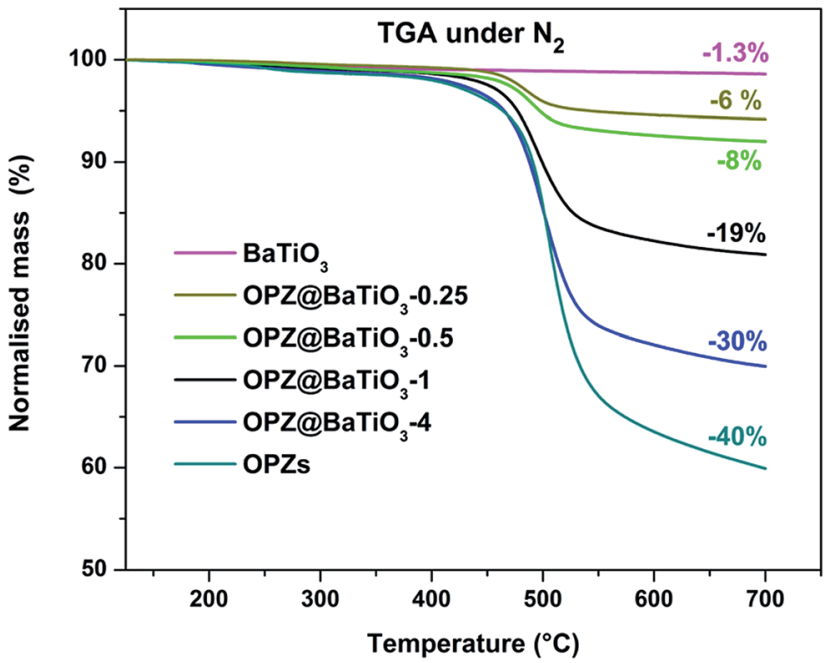

Fig. 4 TGA curves under $\mathrm{N}_{2}$ the $\mathrm{OPZaBaTiO}_{3}$ and the bare $\mathrm{BaTiO}_{3}$ nanoparticles. continued at a slower rate above $500{ }^{\circ} \mathrm{C}$. In order to understand the thermal degradation behaviour, dense OPZs nanospheres were also evaluated under the same conditions; see OPZs in Fig. S1a. $\dagger$ Above $500{ }^{\circ} \mathrm{C}$, a similar behaviour to the OPZ@BaTiO samples was observed, which attributed to the slower decomposition of the inorganic part of the nanospheres with some oxidation (mainly the $\mathrm{P}$ ) taking place simultaneously and

Table 1 Summary of the TGA results of different samples tested under nitrogen and air

\begin{tabular}{|c|c|c|c|}
\hline \multirow{2}{*}{$\begin{array}{l}\text { Atmosphere } \\
\text { Sample name } \\
\text { [shell@core] }\end{array}$} & \multirow{2}{*}{$\begin{array}{l}\text { Nitrogen } \\
\text { Mass loss } \\
(\%)\end{array}$} & \multirow{2}{*}{$\begin{array}{l}\text { Air } \\
\text { Mass loss } \\
(\%)\end{array}$} & \multirow[b]{2}{*}{$\begin{array}{l}\mathrm{BaTiO}_{3} \\
\text { wt } \%\end{array}$} \\
\hline & & & \\
\hline $\mathrm{BaTiO}_{3}$ & 1.3 & 1.6 & 100 \\
\hline $\mathrm{OPZ@BaTiO}_{3}-0.25$ & 6 & 10 & 88 \\
\hline OPZ@BaTiO 3 -0.5 & 8 & 13 & 84.5 \\
\hline OPZ@BaTiO & 19 & 33 & 61 \\
\hline $\mathrm{OPZ@BaTiO}_{3}-4$ & 30 & 56 & 33 \\
\hline OPZ nanospheres & 40 & 84 & 0 \\
\hline${\mathrm{C} @ \mathrm{BaTiO}_{3}-0.25}$ & - & 3.5 & 96.5 \\
\hline $\mathrm{C} @ B a T i O_{3}-0.5$ & - & 7 & 93 \\
\hline $\mathrm{C@BaTiO}_{3}-1$ & - & 21 & 79 \\
\hline $\mathrm{C} @ \mathrm{BaTiO}_{3}-4$ & - & 38 & 62 \\
\hline
\end{tabular}


resulting in a residual mass of $16 \mathrm{wt} \%$. The TGA under air of the bare $\mathrm{BaTiO}_{3}$ particles did not show any oxidation stage, as would be expected since $\mathrm{BaTiO}_{3}$ is already in an oxidized state. However, the OPZ@BaTiO ${ }_{3}-0.25$ and OPZ@BaTiO -0.5 show a slight mass increase at high temperatures which was attributed to the oxidation of the $\mathrm{P}$ atoms. In contrast, the $\mathrm{P}$ oxidation was not evident for the samples with thicker OPZ shells due to the ongoing organic content decomposition at $800{ }^{\circ} \mathrm{C}$, which resulted to negative slopes. According to the above findings, and taking in to account that there is some inorganic residue from the hybrid OPZ shell after TGA under air, the mass percentage of the $\mathrm{BaTiO}_{3}$ in $\mathrm{OPZ} @ \mathrm{BaTiO}_{3}$ can be calculated from eqn (5) where the OPZ residue is $16 \mathrm{wt} \%$ and OPZ weight loss is $84 \mathrm{wt} \%$.

$$
\begin{aligned}
\mathrm{BaTiO}_{3} \mathrm{wt}^{\circ}= & 100 \%-\left(\text { shell@ } \mathrm{BaTiO}_{3} \mathrm{wt} \%\right. \\
& \left.+\frac{\mathrm{OPZ} @ \mathrm{BaTiO}_{3} \mathrm{wt} \% \text { loss } \times \text { OPZ residue } \mathrm{wt} \%}{\mathrm{OPZ} \mathrm{wt} \% \text { loss }}\right)
\end{aligned}
$$

More detailed information for the morphology and structure of the core-shell particles was provided by the TEM micrographs (Fig. 5). The OPZ@BaTiO ${ }_{3}$ samples consisted of multiple $\mathrm{BaTiO}_{3}$ nanoparticles cores covered with the cross-linked OPZ shell of thickness proportional to the monomers' concentration. Thus, the thinnest OPZ shell $(\sim 4 \mathrm{~nm})$ was observed for the OPZ@BaTiO ${ }_{3}-0.25$ (Fig. 5b), while the OPZ@BaTiO ${ }_{3}-4$ had a shell thickness of 40-70 $\mathrm{nm}$ (Fig. 5d). The shell thickness distribution was observed for all the samples which was attributed to the shape irregularity of the $\mathrm{BaTiO}_{3}$ particles and the multiple-nanoparticle core structure as well. The TEM-EDX mapping showed a uniform distribution of all the elements originating from the cross-linked HCCP-BPS network around the $\mathrm{BaTiO}_{3}$ particles (Fig. $\mathrm{S} 2 \dagger$ ). A representative morphology of the core-shell structure after carbonization is shown in Fig. S3a. $\uparrow$ As expected, no graphitic carbon was observed since the OPZ shell, due to its high cross-linking degree, could not undergo graphitization and resulted to a "hard carbon" structure, instead. Additionally, the diffusive diffraction rings shown in the selected area of diffraction (SAED) (Fig. S3b $\dagger$ ), confirmed the amorphous nature or low crystallinity of the carbon shell, while the "spotty" diffraction peaks indicated a polycrystalline $\mathrm{BaTiO}_{3}$ core.

A representative Raman spectra of the $\mathrm{OPZ@BaTiO}_{3}$ is shown in Fig. S4a. $\uparrow$ The characteristic peaks at 729, 1154, 1588 and $3070 \mathrm{~cm}^{-1}$ were assigned to the $\mathrm{C}-\mathrm{S}$, symmetric $\mathrm{O}=\mathrm{S}=\mathrm{O}$, aromatic $\mathrm{C}-\mathrm{C}$ and aromatic $\mathrm{C}-\mathrm{H}$ stretch vibrations, respectively. Additionally, peaks from the $\mathrm{BaTiO}_{3}$ core particles were observed at lower Raman shifts $\left(514,304,248\right.$ and $\left.185 \mathrm{~cm}^{-1}\right)$ of the spectrum. For all the samples the intensities of the OPZ and $\mathrm{BaTiO}_{3}$ peaks were proportional and inversely proportional, respectively, to the shell thickness. The $\mathrm{C} @ B a T i O_{3}$ samples (Fig. S4b $\dagger$ ) showed the presence of the characteristic broad peaks at $1588 \mathrm{~cm}^{-1}$ (G band), $1344 \mathrm{~cm}^{-1}$ (D band), and their second order vibrations at the higher frequency range of the spectra, of hard carbons derived from high temperature pyrolysis. The broadening of the peaks suggested that they consisted of a combination of other peaks and a deconvolution process was required in order to reveal the true carbon structure. The first-order Raman peaks consists of a combination of mixed Gaussian and Lorentzian distributions while the second-order peaks were fitted with Lorentzian-shaped bands. The extent of graphitization was calculated from the $I_{\mathrm{D} 1} / I_{\mathrm{G}}$ ratio, resulting in a value of 1.08 which is representative of carbonaceous materials with a low degree of graphitization (hard carbons) and small graphene sheets with a "house of cards" arrangement. ${ }^{26,27}$
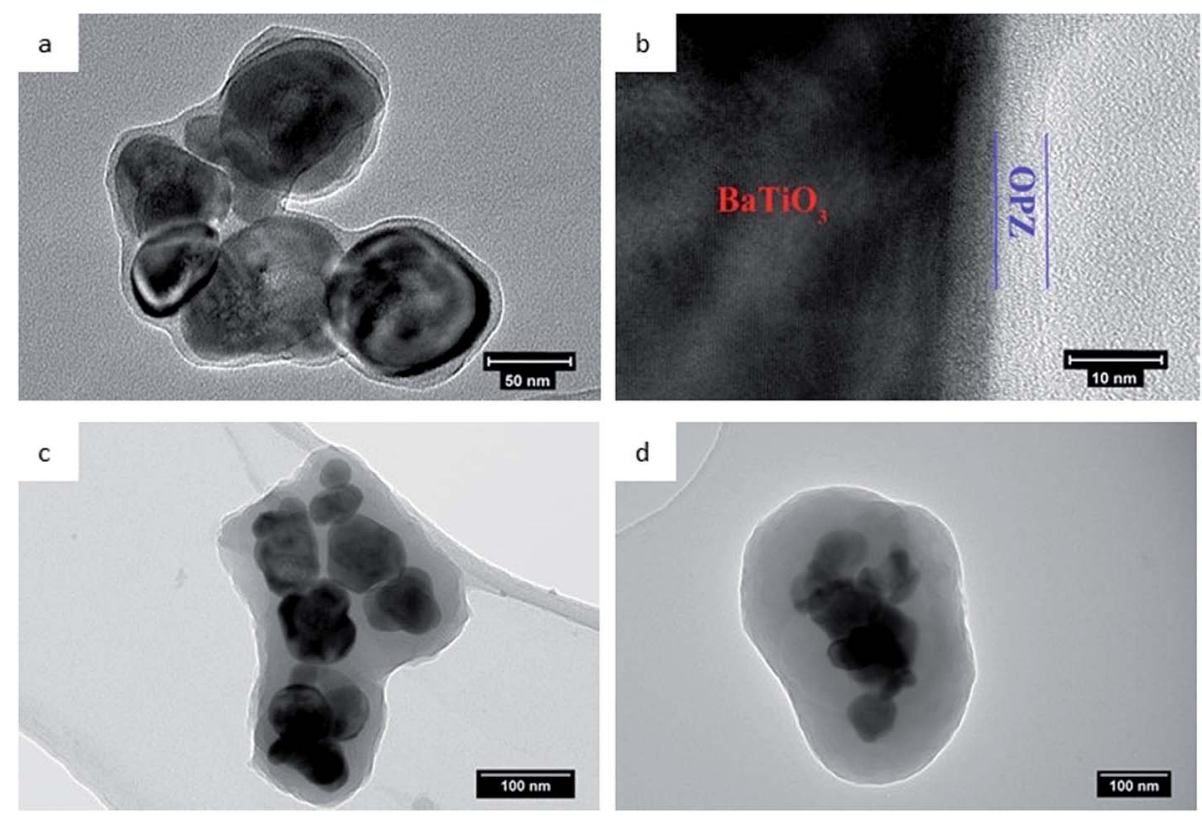

Fig. 5 TEM micrographs of OPZ@BaTiO 3 with (a and b) $0.25: 1$, (c) $1.33: 1$ and (d) $4: 1$ feed ratio. 
The XRD patterns of the OPZ@BaTiO ${ }_{3}$ showed sharp peaks assigned to the cubic crystal structure (paraelectric) of $\mathrm{BaTiO}_{3}$ while the $\mathrm{C} @ \mathrm{BaTiO}_{3}$ showed additionally a broad and intensity below $2 \theta=25^{\circ}$ which was attributed to the hard carbon shell (Fig. S5 $†$ ). Although, the higher dielectric constant of $\mathrm{BaTiO}_{3}$ is originated from the tetragonal crystalline phase usually formed at sintering temperatures $>800{ }^{\circ} \mathrm{C},{ }^{28}$ here, the $\mathrm{OPZ} @ \mathrm{BaTiO}_{3}$ were heat treated as fine powders (not pressed pellets) in order to prevent breakage of the hybrid shell and aggregation at high temperatures. ${ }^{29}$ It is worth noting that, despite the findings from the XRD analysis, the Raman spectra showed a peak at 304 $\mathrm{cm}^{-1}$ which is characteristic of the tetragonal phase of $\mathrm{BaTiO}_{3}$, indicated the mixed crystal structure of the as-received commercial material..$^{30,31}$ The second characteristic peak at $\sim 715 \mathrm{~cm}^{-1}$ of the tetragonal structure, appeared as a shoulder due to the overlapping with the $\mathrm{C}-\mathrm{S}$ vibration at $729 \mathrm{~cm}^{-1}$ (Fig. S4c广).

$\mathrm{N}_{2}$ adsorption-desorption isotherms were used to determine the textural properties of the materials and the representative isotherms from each group of samples are shown in Fig. S6a, $\uparrow$ since all the related samples had similar sorption behaviour. The OPZ@BaTiO ${ }_{3}$ exhibited a Type-III isotherm, typical of nonporous powder materials with a characteristic steep adsorption close to the saturation pressure $\left(>0.9 P / P_{0}\right)$ due to the capillary condensation that takes place in the inter-particle space. The BET specific surface, calculated at the range $0.05-0.30 P / P_{0}$, decreased from 34 to $7 \mathrm{~m}^{2} \mathrm{~g}^{-1}$ area as the shell thickness increased and was attributed to the transformation of the thinshelled irregular particles to more regular thick-shelled particles at a higher monomer feeding ratio. As the OPZ shell transformed to high disordered and heteroatom-doped carbon, a "knee" appeared at the low $P / P_{0}$ region and the $\mathrm{N}_{2}$ adsorbed amount was significantly increased. The isotherm could be assigned as a mixed Type-I isotherm (micropores) and Type-IV (mesopores) due to the presence of micropores in the carbon shell, the pore filling took place before or at the same $P / P_{0}$ range where the multilayer adsorption is usually taking place and a calculation with the BET method, in the traditional range of 0.05-0.35 $P / P_{0}$, resulted in negative $C$ constant. For this reason, an updated BET method was used where the $P / P_{0}$ points are selected in the range of continuously increasing $V\left(1-P / P_{0}\right)$ values. ${ }^{32}$ The contribution of the micropores in the total specific surface area was determined by the $t$-plot method (Carbon Black model) applied in the range 0.01-0.05 $P / P_{0}$. The textural properties of all the samples are summarized in Table S3. $\dagger$ The pore size distribution of the $\mathrm{C} @ \mathrm{BaTiO}_{3}$ samples was obtained from the non-local density functional theory (NLDFT) approach and selecting a model for carbons with a slit pore geometry. As shown in Fig. S5b, $\uparrow$ there are two major peaks at 1.3 and $2.1 \mathrm{~nm}$ (micropores) and a small number of suprapores at 3.3-4.3 nm and mesopores at 4.4-6.4 $\mathrm{nm}$. The microporous structure of the carbon shell was attributed to: (i) degradation and transformation of the organic part of OPZ shell to carbon, (ii) the presence of heteroatoms that escape the shell during carbonization and leave gaps behind, (iii) the size and concentration variety of heteroatoms which introduced more defected structure, and (iv) the "hard-carbon" nature of the formed material.
The porous structures were difficult to observe or identify with TEM analysis, due to the high mixed and disorder structure consisted of with amorphous carbon and small graphitic crystals of random orientations.

XPS analysis was performed in order to examine in more detail the composition of the nanostructures. The survey wide

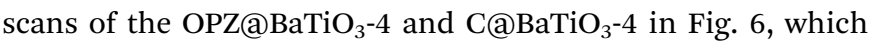
are representative for all the samples, show the presence of the all the elements that are expected to be observed in the $\mathrm{OPZ}$ shell and the carbon shell after the pyrolysis process. The atomic weight percentages were calculated from the core-level scanning of each element and the results are presented in Table S4. $\dagger$ The $\mathrm{P} / \mathrm{S}$ ratio $(\sim 1)$ indicated that the monomeric unit of the cross-linked OPZ structure consisted of one phosphonitrilic trimer connected with three BPS molecules which is in agreement with the initial molar ratio of the monomers $(3: 1)$. It is known from previous reports that nucleophiles with a relative large and non-planar structure take part only in nongeminal substitution of the $\mathrm{P}-\mathrm{Cl}$, thus each $\mathrm{P}$ atom is bonded with only one BPS molecule. ${ }^{33}$ The remaining $\mathrm{P}-\mathrm{Cl}$ could be substituted by any $\mathrm{H}_{2} \mathrm{O}$ molecules that are present in the commercial chemicals (monomers, solvents) and in the atmosphere, forming $\mathrm{P}=\mathrm{O}$ or $\mathrm{P}-\mathrm{OH}$ bonds. A small amount of $\mathrm{Cl}(<1$ at\%) mainly originated from unreacted $\mathrm{P}-\mathrm{Cl}$ and some $\mathrm{Cl}$ with ionic character (TEA $\cdot \mathrm{HCl}$ ) that may be trapped in the structure and its presence, even in a small percentage here, contributed in the formation of the microporous carbon structure during pyrolysis. $^{34}$ The resolved spectra of the high-resolution scans also showed a multi-binding state for all the elements before and after carbonization (Fig. S5 $\dagger$ ). In particular, the $\mathrm{O} 1 \mathrm{~s}$ peak of the OPZ@BaTiO ${ }_{3}$ was resolved in three different peaks with the most significant, at $531.4 \mathrm{eV}$, assigned to the surface aromatic $\mathrm{C}-\mathrm{OH}$ which is important property for a good dispersion in organic solvents and high interaction of these materials in dielectric polymer matrices (PVDF). The absence of $\mathrm{Ba} 3 \mathrm{~d}$ and $\mathrm{Ti}$ $2 \mathrm{p}$ peaks in the $\mathrm{OPZ} @ \mathrm{BaTiO}_{3}-4$ sample is attributed to the thick

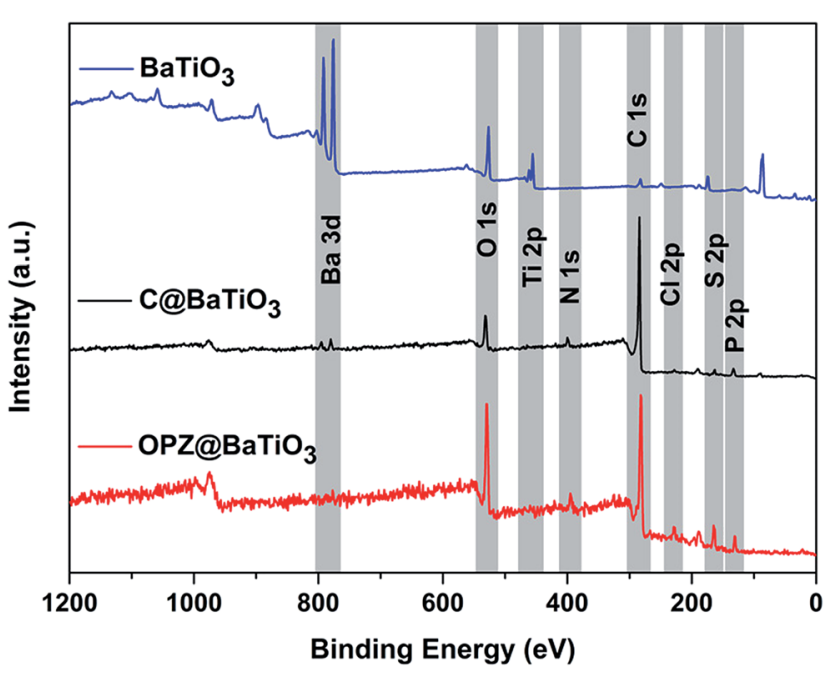

Fig. 6 XPS survey scans of the as received $\mathrm{BaTiO}_{3}$ and the core-shell particles before and after carbonization. 
OPZ shell which is much larger than the penetration depth of the incident beam $(\sim 10 \mathrm{~nm})$.

After the pyrolysis process the converted carbon structure doped with $\mathrm{O}, \mathrm{P}, \mathrm{N}$ and $\mathrm{S}$, showed a significant change of the elements binding states and it is well known that the presence of heteroatoms in carbons significantly alter their electronic as well as their textural properties. ${ }^{35,36}$ The analysis of the $\mathrm{N} 1 \mathrm{~s}$ peak showed the presence of three different components at 398.2, 400.2 and $402.6 \mathrm{eV}$ assigned to pyridinic, pyrrolic/quaternary and oxidized nitrogen which contribute to the electronic properties of the carbon. $\mathrm{P}$ and $\mathrm{S}$ showed a decrease in the number of the different components which is explained by their presence mainly as oxides in the structure that contributes to the formation of the extended microporous structure of the carbon shell ${ }^{23}$ (Fig. S6c-e $\dagger$ ). Among the heteroatoms, S had the lowest at $\%$ while the highest was for $\mathrm{O}$ due to some oxidation that took place during carbonization in the furnace. In contrast to OPZ@BaTiO the survey spectrum of the carbonized samples clearly showed the peaks from the $\mathrm{BaTiO}_{3}$ core particles. Specifically, the spinorbital splitting of the $\mathrm{Ba} 3 \mathrm{~d}$ peak was observed in the $\mathrm{C} @ \mathrm{BaTiO}_{3}$ 4 despite the fact that its shell was thicker than the noncarbonized OPZ@BaTiO ${ }_{3}-1$ (thin shell). This observation was related with two factors: (i) the difference in electronic conductivity between the OPZ (insulator) and $\mathrm{C}$ (conductor) shell so that the exited photoelectrons can "travel" easier through the $\mathrm{C}$ shell. (ii) The lower density of the $\mathrm{C}$ shell allows for deeper penetration of the incident X-ray beam and decreased inelastic energy loss of the extracted photoelectrons.

\subsection{Dielectric properties of the core-shell particles}

Fig. 7 shows the frequency dependent ac conductivity, permittivity, phase angle and dielectric loss for the core-shell powders along with impedance measurements on dense OPZ nano-

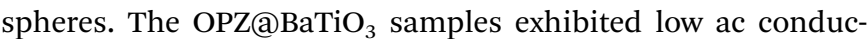
tivity and the linear relationship with frequency in Fig. 7a was a result of the frequency dependences of the admittances of the capacitive regions $(i \omega C)$ that contributed to the conduction paths in these highly insulating materials. This can also be observed in Fig. 7b, whereby in these purely capacitive materials the ac current lagged the ac voltage by $90^{\circ}$; this is a further indication of the dielectric and capacitive nature of the ${\mathrm{OPZ} @ \mathrm{BaTiO}_{3}}$ samples. The dense OPZ nanospheres and the OPZ@BaTiO ${ }_{3}-4$ core-shell particles with the thicker OPZ shells exhibited the lowest ac conductivities (Fig. 7a) and the highest phase angle (Fig. 7b) due to the insulating nature of the OPZ shell.

In contrast, the $\mathrm{C}_{\text {a }} \mathrm{BaTiO}_{3}$ samples had much higher conductivity at low frequencies (DC conductivity) and the conductivity in these core-shell particles was frequency independent (Fig. 7a). Such a response is indicative of conductive behaviour since the admittance of a resistor is simply $1 / R$ and
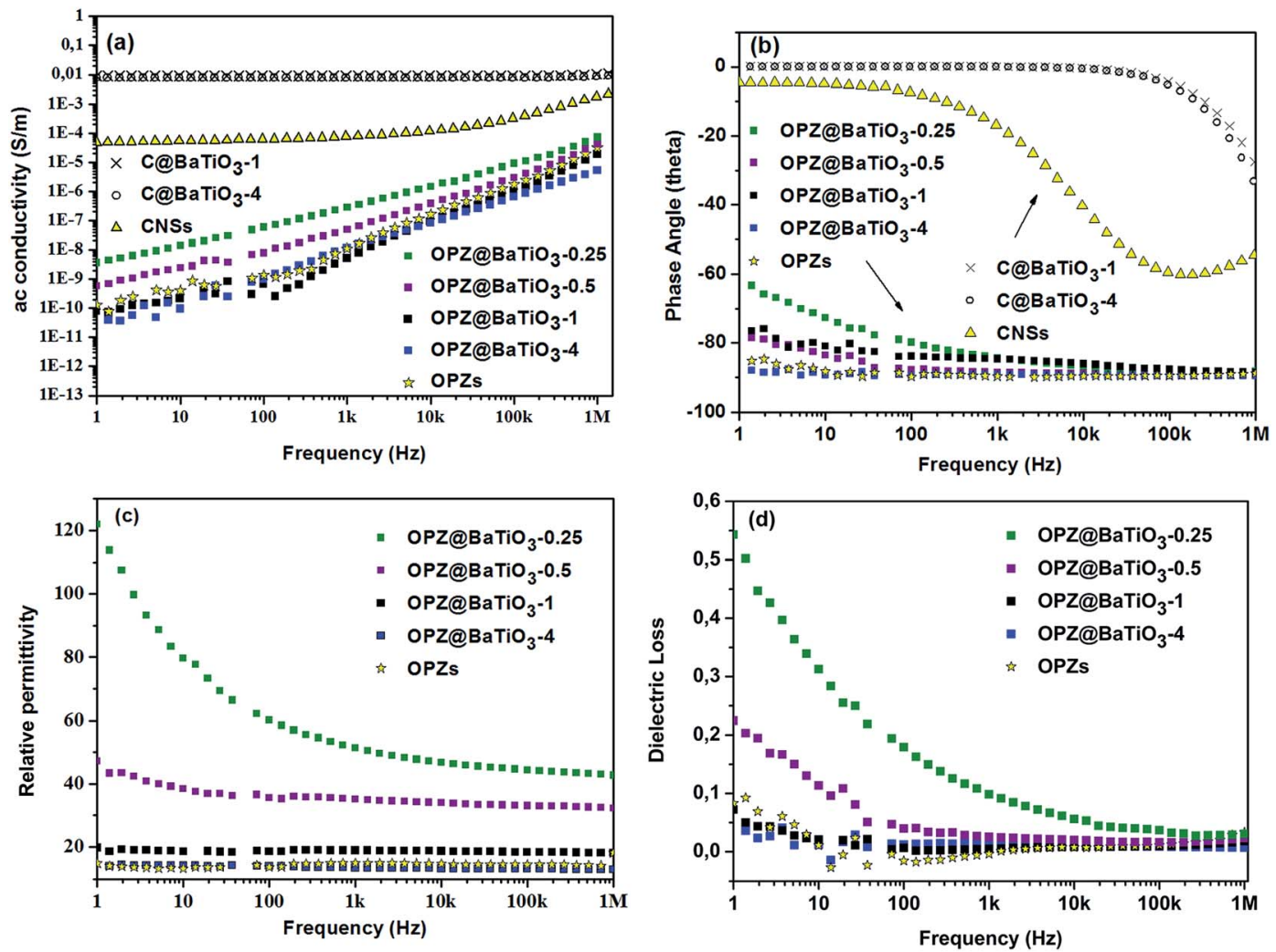

Fig. 7 (a) $\mathrm{AC}$ conductivity of the $\mathrm{OPZ}\left(\mathrm{BBTTO}_{3}\right.$ and $\mathrm{CaBTO}$ samples and comparison with the dense OPZ, (b) phase angle of OPZ(aBaTiO 3 and C ( $B$ BTO samples. Phase angle approaching 0 deg indicates conductive behavior, approaching 90 deg indicates capacitative behavior, (c) relative permittivity of the OPZ@BaTiO 3 samples and comparison with the dense OPZ nanospheres. (d) Dielectric loss of the OPZ@BaTiO 3 samples and comparison with the dense OPZ nanospheres. 
this can also be seen in Fig. $7 \mathrm{~b}$ where the phase angle approaches $0^{\circ}$ where current and voltage are now in phase.

Fig. 7c shows the frequency dependent relative permittivity of the OPZ@BaTiO ${ }_{3}$ materials. The relative permittivity increased as the OPZs shell becomes thinner and the contribution of $\mathrm{BaTiO}_{3}$ to the relative permittivity is more effective; this can also be seen in Table 2. The observed frequency dependence of the permittivity in Fig. 7c, at low frequencies, was more pronounced in the samples with a thin OPZ layer and has been shown to be related to the presence of conductivity in the material. ${ }^{37}$ This can be seen in Fig. $7 d$ where the samples with higher dielectric loss exhibited the stronger frequency dependence at low frequencies (Fig. 7c). At higher frequencies the admittance of the capacitive regions $(i \omega C)$ increased and ac currents flowed through the capacitative (insulating) regions of the material and the influence of conductivity on permittivity and dielectric loss was therefore reduced; see Fig. $7 \mathrm{c}$ and $\mathrm{d}$ where the responses are almost frequency independent above $10^{3} \mathrm{~Hz}$. The $\mathrm{C} @ \mathrm{BaTiO}_{3}$ samples are not shown since they exhibit high ac conductivity and the permittivity is not relevant.

Since the bare OPZ nanospheres showed very low conductivity and frequency independent permittivity, the conductivity was likely to originate from the $\mathrm{BaTiO}_{3}$. Indeed, the high resolution XPS of the "as received" $\mathrm{BaTiO}_{3}$ showed the presence of $\mathrm{BaCO}_{3}$ as it can be seen from the deconvolution of the $\mathrm{C} 1 \mathrm{~s}, \mathrm{Ba} 3 \mathrm{~d}_{5 / 2}$ and $\mathrm{O}$ 1s peaks (Fig. $\mathrm{S} 7 \dagger$ ). The presence of carbonate, which contributes to the conductivity, is common for the perovskite materials and it is originated from the synthetic procedures as well as from the reaction with the atmospheric $\mathrm{CO}_{2} \cdot{ }^{38-40}$ Therefore, the nanoparticles with a thin shell showed higher dielectric losses compared to the thick OPZ shells which provide more insulating characteristics. A decreasing permittivity and dielectric loss with increasing frequency, has been observed for thin films of $\mathrm{BaTiO}_{3}$-polymer core-shell particles with even lower ac conductivities than reported here. ${ }^{\mathbf{4 1 , 4 2}}$ In this work the measurements were conducted on pressed powders and the presence of lowpermittivity air between the inter-particle spaces can lower the measured effective permittivity and give some inaccuracy.

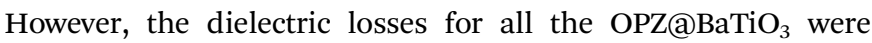
surprisingly low. The above results in Fig. 7 suggest that the electrical properties of organophosphazene-decorated $\mathrm{BaTiO}_{3}$ particles, can therefore be readily tailored from highly insulating (capacitive) and low dielectric loss $\left(\mathrm{OPZ} @ \mathrm{BaTiO}_{3}\right)$ to electrically conducting (C@BaTiO ${ }_{3}$ ), by varying the shell thickness and chemical structure. The above properties could make these materials good candidates as hybrid fillers $\left(\mathrm{OPZ} @ \mathrm{BaTiO}_{3}\right)$ in

Table 2 Relative permittivity and loss at $1 \mathrm{kHz}$

\begin{tabular}{llll}
\hline $\begin{array}{l}\text { Sample name } \\
\text { [shell@core] }\end{array}$ & $\begin{array}{l}\text { Relative } \\
\text { permittivity } \varepsilon^{\prime}\end{array}$ & $\begin{array}{l}\text { Dielectric } \\
\text { loss }\end{array}$ & $\begin{array}{l}\mathrm{BaTiO}_{3} \\
\mathrm{wt} \%\end{array}$ \\
\hline OPZ@BaTiO $_{3}-0.25$ & 51 & 0.098 & 88 \\
OPZ@BaTiO $_{3}-0.5$ & 35 & 0.025 & 85 \\
OPZ@BaTiO $_{3}-1$ & 19 & 0.005 & 61 \\
OPZ@BaTiO $_{3}-4$ & 13 & 0.015 & 33 \\
OPZ nanospheres & 15 & 0.006 & 0
\end{tabular}

ferroelectric polymer nanocomposites for the improvement of the high dielectric loss of the polymer matrix, as well as active fillers (C@BaTiO ${ }_{3}$ ) in dielectric percolative composites.

\section{Conclusions}

This paper presented a facile and novel method to functionalise $\mathrm{BaTiO}_{3}$ nanoparticles with an organophosphazene insulating shell at ambient conditions and within a short timescale. It was demonstrated that the OPZ shell thickness can be readily controlled by adjusting the feeding ratio of the monomers to $\mathrm{BaTiO}_{3}$. The electrically insulating core-shell OPZ@BaTiO ${ }_{3}$ nanoparticles could also be transformed into electrically conducting carbon coated $\mathrm{BaTiO}_{3}$ nanoparticles with well-preserved morphologies by a simple carbonization treatment. This work provides a facile and cost-effective method to generate novel coreshell nanoparticles with well-defined shell structures and properties that range from electrically insulating (OPZ) to highly conductive (carbon). The resultant products can be readily applied as active materials in electrochemical energy storage applications.

\section{Acknowledgements}

G. S. P. acknowledges the PhD research funding sponsored by WMG at the University of Warwick and Dr Marc Walker (Physics) for helping with the XPS measurements and Dr Geoff West (WMG) for TEM-EDX. C. Wan acknowledges the funding from EPSRC-iCASE award 6560. C. B. acknowledges funding from the European Research Council under the European Union's Seventh Framework Programme (FP/2007-2013)/ERC Grant Agreement no. 320963 on Novel Energy Materials, Engineering Science and Integrated Systems (NEMESIS).

\section{References}

1 A. J. Lovinger, Science, 1983, 220, 1115-1121.

2 Q. M. Zhang, Science, 1998, 280, 2101-2104.

3 S. Cho, J. S. Lee and J. Jang, Adv. Mater. Interfaces, 2015, 2, 1500098.

4 K. Yang, X. Huang, M. Zhu, L. Xie, T. Tanaka and P. Jiang, ACS Appl. Mater. Interfaces, 2014, 6, 1812-1822.

5 T. Zhou, J. W. Zha, R. Y. Cui, B. H. Fan, J. K. Yuan and Z. M. Dang, ACS Appl. Mater. Interfaces, 2011, 3, 2184-2188.

6 P. Kim, N. M. Doss, J. P. Tillotson, P. J. Hotchkiss, M. J. Pan, S. R. Marder, J. Li, J. P. Calame and J. W. Perry, ACS Nano, 2009, 3, 2581-2592.

7 X. Zhang, Y. Ma, C. Zhao and W. Yang, Appl. Surf. Sci., 2014, 305, 531-538.

8 N. Jia, Q. Xing, X. Liu, J. Sun, G. Xia, W. Huang and R. Song, J. Colloid Interface Sci., 2015, 453, 169-176.

9 X. Zhang, S. Wei, N. Haldolaarachchige, H. A. Colorado, Z. Luo, D. P. Young and Z. Guo, J. Phys. Chem. C, 2012, 116, 15731-15740.

10 K. Yang, X. Huang, Y. Huang, L. Xie and P. Jiang, Chem. Mater., 2013, 25, 2327-2338.

11 M. Zhu, X. Huang, K. Yang, X. Zhai, J. Zhang, J. He and P. Jiang, ACS Appl. Mater. Interfaces, 2014, 6, 19644-19654. 
12 L. Xie, X. Huang, Y. Huang, K. Yang and P. Jiang, J. Phys. Chem. C, 2013, 117, 22525-22537.

13 A. C. Patsidis, K. Kalaitzidou, D. L. Anastassopoulos, A. A. Vradis and G. C. Psarras, J. Chin. Adv. Mater. Soc., 2014, 2, 207-221.

14 Z. M. Dang, L. Wang, Y. Yin, Q. Zhang and Q. Q. Lei, Adv. Mater., 2007, 19, 852-857.

15 Z.-M. Dang, S.-H. Yao, J.-K. Yuan and J. Bai, J. Phys. Chem. C, 2010, 114, 13204-13209.

16 J.-K. Yuan, S.-H. Yao, Z.-M. Dang, A. Sylvestre, M. Genestoux and J. Bai, J. Phys. Chem. C, 2011, 115, 5515-5521.

17 Y. Feng, W. L. Li, J. P. Wang, J. H. Yin and W. D. Fei, J. Mater. Chem. A, 2015, 3, 20313-20321.

18 Y. Zhu, X. Huang, W. Li, J. Fu and X. Tang, Mater. Lett., 2008, 62, 1389-1392.

19 L. Zhu, Y. Xu, W. Yuan, J. Xi, X. Huang, X. Tang and S. Zheng, Adv. Mater., 2006, 18, 2997-3000.

20 P. Zhang, X. Huang, J. Fu, Y. Huang, Y. Zhu and X. Tang, Macromol. Chem. Phys., 2009, 210, 792-798.

21 S. Yang, C. Cao, Y. Sun, P. Huang, F. Wei and W. Song, Angew. Chem., 2015, 54, 2661-2664.

22 J. Fu, Q. Xu, J. Chen, Z. Chen, X. Huang and X. Tang, Chem. Commun., 2010, 46, 6563-6565.

23 G. Pappas, S. Ferrari, X. Huang, R. Bhagat, D. Haddleton and C. Wan, Materials, 2016, 9, 35.

24 V. K. LaMer and R. H. Dinegar, J. Am. Chem. Soc., 1950, 72, 4847-4854.

25 J. B. Ledeuil, A. Uhart, S. Soule, J. Allouche, J. C. Dupin and H. Martinez, Nanoscale, 2014, 6, 11130-11140.

26 A. Sadezky, H. Muckenhuber, H. Grothe, R. Niessner and U. Pöschl, Carbon, 2005, 43, 1731-1742.

27 J. R. Dahn, W. Xing and Y. Gao, Carbon, 1997, 35, 825-830.

28 P. K. Dutta, R. Asiaie, S. A. Akbar and W. Zhu, Chem. Mater., 1994, 6, 1542-1548.
29 C. W. Beier, M. A. Cuevas and R. L. Brutchey, Langmuir, 2010, 26, 5067-5071.

30 M. B. Smith, K. Page, T. Siegrist, P. L. Redmond, E. C. Walter, R. Seshadri, L. E. Brus and M. L. Steigerwald, J. Am. Chem. Soc., 2008, 130, 6955-6963.

31 M. El Marssi, F. Le Marrec, I. A. Lukyanchuk and M. G. Karkut, J. Appl. Phys., 2003, 94, 3307.

32 M. Thommes, K. Kaneko, A. V. Neimark, J. P. Olivier, F. Rodriguez-Reinoso, J. Rouquerol and K. S. W. Sing, Pure Appl. Chem., 2015, 87, 1051-1069.

33 R. De Jaeger and M. Gleria, Prog. Polym. Sci., 1998, 23, 179276.

34 Y. Zhu, H. Cui, X. Meng, J. Zheng, P. Yang, L. Li, Z. Wang, S. Jia and Z. Zhu, ACS Appl. Mater. Interfaces, 2016, 8, 6481-6487.

35 X. Wang, G. Sun, P. Routh, D. H. Kim, W. Huang and P. Chen, Chem. Soc. Rev., 2014, 43, 7067-7098.

36 G. Hasegawa, T. Deguchi, K. Kanamori, Y. Kobayashi, H. Kageyama, T. Abe and K. Nakanishi, Chem. Mater., 2015, 27, 4703-4712.

37 D. P. Almond and C. R. Bowen, Phys. Rev. Lett., 2004, 92, 157601.

38 C. Miot, E. Husson, C. Proust, R. Erre and J. P. Coutures, J. Eur. Ceram. Soc., 1998, 18, 339-343.

39 M. Wegmann, L. Watson and A. Hendry, J. Am. Ceram. Soc., 2004, 87, 371-377.

40 M. D. C. B. López, G. Fourlaris, B. Rand and F. L. Riley, J. Am. Ceram. Soc., 1999, 82, 1777-1786.

41 L. Xie, X. Huang, C. Wu and P. Jiang, J. Mater. Chem., 2011, 21, 5897.

42 L. Xie, X. Huang, Y. Huang, K. Yang and P. Jiang, ACS Appl. Mater. Interfaces, 2013, 5, 1747-1756. 